\title{
Investigation of the order -disorder phase transition series in AuCu by in-situ temperature XRD and mechanical spectroscopy
}

\author{
Imene Lamiri ${ }^{1,3,4^{*}}$, David Martinez Blanco ${ }^{2}$, Mohammed S.M.Abdelbaky ${ }^{3}$, Daniele Mari ${ }^{4}$, Djamel Hamana ${ }^{1}$ and Santiago Garcia \\ Granda $^{3}$ \\ 1-Research Unit Materials Science and Applications, Mentouri University and Ecole National Polytechnique de Constantine, \\ 25000 Constantine, Algeria. \\ 2- Scientific-Technical Services, University of Oviedo, 33006 Oviedo, Spain., 33006 Oviedo, Spain. \\ 3- Department of Physical and Analytical Chemistry, University of Oviedo-CINN, 33006 Oviedo, Spain. \\ 4- IPHYS, Ecole Polytechnique Fédérale de Lausanne, Station 3, 1015 Lausanne, Switzerland
}

\section{Abstract}

In-situ temperature XRD and mechanical spectroscopy were applied to study phase transitions in AuCu alloy from the disordered FCC state (A1). The phase transition temperature intervals were investigated upon heating and cooling using a rate of $1 \mathrm{~K} / \mathrm{min}$ in order to be thoroughly determined. The following sequences of phase transitions were recorded upon continuous heating: $A 1->A u C u l->A u C u l l->A 1$ and on subsequent cooling: A1 ->A1+AuCul+AuCull $->A u C u l+A u C u l l ~->A u C u l$. This transition sequences determine also temperature dependency of elastic and anelastic properties. Mechanical spectroscopy using a forced torsion pendulum shows a peak due to antiphase boundaries motion characterizing the orthorhombic phase AuCull and a transient peak with the that appears in the tetragonal phase AuCul together with the classical Zener peak in the A1 phase.

\section{Introduction}

It is well known that order-disorder transitions produce significant effects on the properties of alloys [1, 2]. AuCu alloys appear to be a testing ground for different approaches and models that are aimed at describing phase changes in stoichiometric alloys [1, 3]. A pioneering in-situ work on Au-Cu alloy is that of Pasheley et al. on thin evaporated films [4,5]. Phase transitions in AuCu system have been investigated using several techniques: micro hardness [6], acoustic emission and optical cinematography $[7,8]$. The study of phase transitions in quenched $\mathrm{Au}-\mathrm{Cu}$ alloys under different annealing conditions have been reported in several studies [2, 9-12]. Sometimes, results and interpretations related to temperature intervals and kinetics 
of phase transitions are quite divergent $[10,13,14]$ but, in any case, binary $\mathrm{AuCu}$ system presents two successive solid-solid transformations (between a disordered A1 fcc phase and a long period orthorhombic AuCull phase together with a tetragonal AuCul phase $[3,15]$.

The work of Beaunnaux et al. [10] represents the last and most general investigation of phase transitions in AuCu alloy, using in-situ temperature controlled TEM. They have shown that different contradictions appear in previous studies and concluded that the in-situ TEM study presents some experimental difficulties. Therefore, these authors concluded that an in-situ XRD study would be necessary. Furthermore, mechanical spectroscopy has recently shown as a new approach to understanding the order-disorder phase transition [13, 16-19]. Important works have been devoted to the mechanical behavior [25] of gold alloys related to their industrial uses [18-24, 26, 27]. In fact, the re-arrangement of $\mathrm{Au}$ and $\mathrm{Cu}$ atoms can be monitored by $\mathrm{Cu}$ diffusion and in particular by the re-orientation of $\mathrm{Cu}$ pairs or clusters in the cell, which produces a characteristic peak called the Zener peak [18, 19]. In 1947, Zener first described this anelastic phenomenon, which gives rise to a thermally activated mechanical loss peak [25]. Under the application of an external stress, atoms locally rearrange, thereby minimizing the strain energy. Stress induced directional ordering causes energy dissipation, which is manifested by a damping peak.

In this paper, we used in-situ $X$ ray diffraction to monitor the temperature intervals and kinetics of phase transitions in annealed and quenched AuCu (binary 18-carat) system, during the complete thermal cycle upon heating and cooling. To the best of our knowledge this is the first such study on this alloy. The paper mainly focuses on phase transformation in bulk sample from the disordered state A1. Direct observation of the phase transformations starting from the metastable phase $A 1$ is also a peculiarity of this study. The purpose of this research is to show how these transitions may affect the mechanical properties by measuring both the internal friction and mechanical shear modulus in temperature scanning tests performed at the same conditions as the x-ray diffraction. 


\section{Materials and methods}

The Au-Cu sample was fabricated by induction melting under vacuum. High purity raw materials (Au: 99.999\%, Cu: $99.999 \%$ ) have been used for its production. The Electron Probe Microanalysis shows that the chemical composition of the ingots was Au-25.14.wt \%Cu (Au-51Cu hereafter), with an accuracy of $0.2 \%$. A disc of $10 \mathrm{~mm}$ of diameter and $0.5 \mathrm{~mm}$ of thickness was prepared from the ingots for the in-situ XRD study. The sample was homogenized at $973 \mathrm{~K}$ for 2 hours, under primary vacuum ( $\left.1 \times 10^{-3} \mathrm{mbar}\right)$, followed with water quenching in order to obtain the disordered state A1 at room temperature (RT). The samples were polished with diamond paste to remove impurities or deposition on the sample surface before the analysis.

In situ X-ray thermo-diffraction was performed inside an Anton Pear HTK $1200 \mathrm{~N}$ chamber between 298 and $973 \mathrm{~K}$ using a rate of $1 \mathrm{~K} / \mathrm{min}$ under secondary vacuum ( $\left.1 \times 10^{-5} \mathrm{mbar}\right)$. This platform is mounted on the goniometer of a Panalytical XPERTPRO diffractometer, which uses copper $K_{\alpha}$ radiation (1.5418 $\AA$ ) and a PIXcel detector to record diffraction patterns measured in sweep mode. The disk is fixed on the top of an alumina rod with a diameter of $15 \mathrm{~mm}$. Suitably fixed slits and masks are selected to illuminate only the surface of the sample. NIST standard reference material SRM 660 a was used to determine precisely angle and/or height errors on the equipment and sample position. Besides, two equivalent experiments have been carried out over a large diffraction angle range (from 20 to $90^{\circ}$ on 2Theta) and a short diffraction angle range (from 29 to $42^{\circ}$ on 2Theta) in order to establish the type and temperatures of each phase transformations respectively. The heating and cooling rate was $1 \mathrm{~K} / \mathrm{min}$ and one pattern was acquired every $10 \mathrm{~min}$ for the large diffraction angle scans and repeated every $1.1 \mathrm{~min}(1 \mathrm{~min} 6 \mathrm{sec})$ for the short diffraction angle scans. Finally, X-ray diffraction patterns have been analyzed by the Le-Bail method [28] implemented in the Fullprof Suite package [29], allowing us to settle the parameters of every peak.

Measurements of internal friction and of dynamic shear modulus were performed in an inverted torsion pendulum as a function of temperature. Temperature scanning spectra were obtained in forced mode at a frequency of $1 \mathrm{~Hz}$ and $0.5 \mathrm{~Hz}$ during heating/cooling at the rate of $1 \mathrm{~K} / \mathrm{min}$ under vacuum. 
The strain amplitude was $5 \times 10^{-5}$. The samples had dimensions of $20 \times 4 \times 0.3 \mathrm{~mm}^{3}$. The phase lag $\delta$ between stress and strain is directly measured and can be related to the mechanical loss, $\tan (\delta)$. The dynamic shear modulus, $M$, at the frequency, $f$, is obtained from the ratio of stress and strain amplitude [24].

\section{Results}

Upon continuous heating, an optimum heating rate was chosen in order to evidence ordering from the quenched state [13]. A heating rate of $1 \mathrm{~K} / \mathrm{min}$ has been chosen. Long diffraction scans, with rate of one pattern taken every $10 \mathrm{~K}$ (1pattern/10min), have been performed to well distinguish the characteristic peaks of every phase since the highest peak of the disordered cubic A1 overlaps with the related peaks of the tetragonal AuCul and the orthorhombic AuCull. On the other hand, scans performed in the short diffraction angle range, with a rate of one pattern taken per $1 \mathrm{~K}$ (precisely 1 pattern $/ 1.1 \mathrm{~min}$ ), have been used to determine the precise temperatures of the phase transitions.

Fig.1. shows the RTX-Ray patterns measured in the initial state (annealed and water quenched) (a) and in the final state after a heating-cooling cycle (b). All Bragg peaks observed in the initial state belong to reflections of the disordered cubic A1 structure although small tetragonal AuCul ordered phase peaks are present. They appear as humps in Fig. 1a at low diffraction angles, which suggests that the AuCul phase grain size is very small. At the end of the heating/cooling cycle, a single microcrystalline ordered tetragonal AuCul is obtained.

Fig. 2a and Fig. S.1 present the 3D plots of the XRD measurements upon heating and cooling for the large diffraction angle scans and for the short diffraction scans, respectively. Three phase transitions can be discerned upon heating as follow: A1 AuCul-AuCull-A1, and two upon cooling: A1-AuCul+AuCull-AuCul. Moreover, in every transition there is an interval of temperatures where two phases coexist. The exact temperatures of every phase transition are presented in Table 1 mainly obtained by analyzing the short diffraction scan data. However, the phase transition temperature upon heating that correspond to the onset of the tetragonal AuCul and the total disappearance of the cubic A1 (i.e. the presence of the pure tetragonal 
AuCul) were extracted not only from short diffraction angles spectra, but also from the long diffraction angles spectra shown in Fig. 3.

Fig.4 and Fig.S.2 show the intensity behaviors of the selected peaks of every phase during the heating/cooling cycle, where data have been extracted from the fitting of the short and large diffraction angle range scans respectively. The intensity of (111) A1 peak upon heating increases abruptly (up to 50\%) in two scans i.e. two degrees before decreasing continuously from 408K with the onset of (101) AuCul peak and the increase of (100) AuCul peak. The (101) AuCul peak decreases before stabilization with the net presence of AuCul phase. In Fig.S.2, the data have been extracted from the large diffraction angle range scans. The intensity of (220) A1 (Fig.S.2 (a)) decreases remarkably upon heating at around $368 \mathrm{~K}$ followed by a second decrease with the onset of (200) AuCul peak. Fig.4 and Fig.S.2 emphasize the sequences of the phase transitions and the transition temperatures upon heating and cooling. The phase transition temperatures are referred with dotted lines. After that, the temperature dependence of peak intensities are correlated with different phase transitions.

On the other hand, the phase transitions in $\mathrm{Au}-51 \mathrm{Cu}$ system show a noticeable difference in thermal expansion coefficients of different phases in terms of normalized volume of the unit cell of the corresponding phases per atom (Fig.S.3) and in terms of total normalized volume (Fig.5). The normalized unit cell volumes have been calculated from fitting XRD patterns of the large diffraction angles scans, where, the obtained unit cell of every phase has been divided by the corresponding number of atoms per cell then used to obtain the normalized volume. To obtain the total normalized volume, $\mathrm{V}_{\mathrm{T}}$, we have used the relation (1).

$$
V_{T}=V_{\alpha} \cdot f_{\alpha}+V_{\beta} \cdot f_{\beta}+\ldots . .
$$

being $\operatorname{V}_{(\alpha, \beta, \ldots)}$ and $\operatorname{fr}(\alpha, \beta, \ldots)$ the normalized volume and fraction for the phases $\alpha, \beta, \ldots$

In both figures, a noticeable decrease of the normalized unit cell volume of $A 1$ phase is manifested in two steps, firstly at the onset of the AuCul phase and then continuously until the total disappearance of $\mathrm{A} 1$. Above $550 \mathrm{~K}$, the normalized unit 
cell volume corresponding to AuCul increases homogenously with respect to the temperature, until the temperature of the second transition (AuCul - AuCull) whereas a noticeable expansion is observed when the AuCull phase appears. A second expansion occurs upon the third transition (AuCull - A1). Upon cooling, a noticeable contraction accompanies the ordering transition at the passage from $A 1$ to $\mathrm{AuCul}+\mathrm{AuCull}$; followed by an expansion when only the AuCul phase remains. Fig. S.4 exhibits the evolution of phase fractions upon heating and cooling.

This dilatometric behavior of the phase transitions at the atomic level obtained for the analysis of XRD patterns is in a good agreement with the macroscopic expansion obtained by means of a dilatometer (Fig.S.5)

Fig. 6 shows the temperature dependence of the internal friction (TDIF) and the elastic modulus (TDEM) of the AuCu alloy obtained with the disordered phase A1 as a starting point. Two frequencies have been used: $1 \mathrm{~Hz}$ (top) and $0.5 \mathrm{~Hz}$ (bottom). Three peaks are visible on both heating curves (red lines), denoted by $P_{1}, P_{z}$ and $P_{A P B}$ as temperature increases. First, the $\mathrm{P}_{1}$ peak appears at the same temperature as the first phase transition shown in the structural study (A1 - AuCul). Then, the Pz peak arises as a broad bump, which spreads over the range where AuCul is observed and has a maximum at the temperature of the AuCul->AuCull phase transition. Finally, the $P_{A P B}$ appears as a sharp spike that is cut off by the transformation into the disordered phase A1. The shear modulus presents dip and shoulders that are synchronized with each internal friction peak. Concerning the frequency, the thermally activated peak $\mathrm{Pz}$ widens toward low temperatures when the frequency decreases but $\mathrm{PAPB}_{\mathrm{AP}}$ remains at the same temperature. On the other hand, the two cooling curves (blue lines) for each frequency have a similar shape and both contain two peaks, the peak $\mathrm{Pz}$ and the peak $\mathrm{PGB}_{\mathrm{GB}}$ that is probably due to a grain boundary sliding. The position of $\mathrm{Pz}$ is almost the same and it becomes sharper at low frequency $(0.5 \mathrm{~Hz})$ where its maximum gets closer to the temperature of the phase transition. The shear modulus upon cooling shows the obvious increase corresponding to the relaxation peak Pz. However, superimposed to this appear some fluctuations that coincide with the region of co-existence of the AuCul and AuCull phases. Coming from the high temperatures, the rapid increase of the Zener 
peak intensity coincides with an increase of the shear modulus curve. When the Zener peak falls down, a steady increase in the shear modulus is observed. Finally, it is worth to notice that the position of the $\mathrm{Pz}$ relaxation peak, is not the same upon heating and cooling.

\section{Discussion}

\subsection{Heating}

In-situ XRD temperature sweeps produce the most accurate picture of the phase transitions in the studied material. However, we can notice that in many cases different phases coexist in a metastable state. The very first transition from the disordered A1 phase to the ordered AuCul needs to be carefully analyzed since, from the beginning of the experiment, AuCul nanograins are present as a result of quenching. Thus, while the intensity of (100) AuCul peak shows a constant value from RT to $408 \mathrm{~K}$, corresponding to the constant quantity of the tetragonal AuCul nanograins, we cannot determine the intensity of the (101) AuCul peak because of the overlapping with the main peak (111) of the cubic phase A1. So, assuming a constant quantity of the tetragonal AuCul nanograins, its intensity is presented as a dotted red line (Fig.4(a)) from RT to 408K. Close to this point (exactly two degrees before), the intensity of the peak (111) of the A1 phase shows a sudden increase in the intensity, which may be due to the coalescence of the disordered crystalline cubic domains. Therefore, the thermally induced decrease of vacancy concentration, generated in the supersaturated phase A1 after quenching, may lead to some dislocation relaxation that sharpens the diffraction peaks and produces the corresponding raise of intensities too. After that, the interval from 408 to $538 \mathrm{~K}$ shows a gradual decrease in the intensity of the (111) A1 peak, which is associated with the corresponding intensity increase observed for the (100) and (101) peaks of AuCul phase (Fig.4(a)). Nevertheless, the assessment of the transition temperature from A1 to AuCul upon heating is visualized at high diffraction angles in the large range scans, while the overlap between (220) A1 and (200) or (212) broad reflections is better resolved in the short range scan (Fig.3). In contrast, the onset of the orthorhombic phase AuCull upon heating, which starts at $637 \mathrm{~K}$ and ends with pure AuCull at $657 \mathrm{~K}$, and the nucleation of the cubic A1 from AuCull (beginning at $677 \mathrm{~K}$ 
and finishing at $685 \mathrm{~K}$ ) are accurately determined from the data of short angle scans (Fig.3). In the region where AuCul is growing to the detriment of $\mathrm{A1}$, a volume contraction proceeds in two steps (Fig.5(a), Fig.S.3(a) and Fig.S.4(a)) and an inflection point around $458 \mathrm{~K}$ is observed. The same effect is noticeable in the macroscopic dilatometer measurements (Fig.S.5).

On the other hand, the internal friction and shear modulus spectra give a better understanding of the defect diffusion or rearrangement processes that occur in the material. In Fig.6, the first phase transition A1-AuCul is visible through the $\mathrm{P}_{1}$ peak that reveals a split shape, which can be explained by the ordering reaction that precedes the A1-AuCul transition as reported previously 30]. What is more important, however, is the modulus variation. Despite the "jumpy" shape of the curve that demonstrates that the transformation probably occurs by successive bursts in different parts of the sample, we have noticed that the transformation always shows a decrease of the modulus followed by an increase. Therefore, one may suppose that the transformation needs some softening of the elastic constants to be triggered. At about $630 \mathrm{~K}$, a maximum is always observed but it is clearly more visible at $0.5 \mathrm{~Hz}$. According to the earlier studies [23], this maximum should be attributed to a Zener relaxation that appears in the ordered phase AuCull and disappears abruptly when such phase transform to the disordered phase $\mathrm{A} 1$ takes place. As demonstrated by Henning [19], the Zener relaxation can only occur if the $\mathrm{Au}$ and $\mathrm{Cu}$ have the possibility to exchange. The presence of the Zener peak in the AuCull ordered phase indicates that the state of atomic order is not perfect. Indeed, due to the non-perfect stoichiometric composition of the alloy, some substitutional disorder must exist i.e. Au atoms occupy sites on the $\mathrm{Cu}$ sublattice [19].The temperature range, which corresponds to the presence of the pure ordered phase AuCull, is characterized by the PAPB peak that may be the antiphase boundaries relaxation peak. Such peak disappears with the onset of the cubic A1. According to refs.[10, 31], the AuCul and AuCull phases are characterized by the presences of twins, which create PAPB. However, Tkalcec et al. [27 suggested a better explanation. They showed that two different peaks can appear: a twin boundaries peak that appears in AuCul and a peak in AuCull that they attribute to the movement of antiphase boundaries. In the present study, the peak PAPB appears in the AuCull region and it is more likely due to 
the presence of the antiphase boundaries. The spectra recorded at a frequency of $0.5 \mathrm{~Hz}$ allow a better discrimination of the phenomena. The Zener peak, which is thermally activated, appears at a lower temperature.

\subsection{Cooling}

The phase transition sequences from high temperature to RT shown by XRD are as follows: $\mathrm{A} 1->\mathrm{A} 1+\mathrm{AuCull}+\mathrm{AuCul}->\mathrm{AuCull}+\mathrm{AuCul}->\mathrm{AuCul}$. From short range diffraction angle scans, there is a small temperature range $(649-645 \mathrm{~K})$ where the three phases A1+AuCul+AuCull coexist (Table1, Fig.3 and Fig.4). From 644 to $604 \mathrm{~K}$, the concomitant presence of only two phases, AuCul and AuCull, can be deduced from the intensities of the (019) and (0111) reflections of AuCull (see Fig.4(b)). By cooling strictly below 604K, only the pure tetragonal AuCul is present. The appearance of order upon cooling down is associated with a noticeable contraction (Fig. 5(b) and Fig.S.3(b))

Upon the cooling the peak $\mathrm{Pz}$ clearly starts only when the phase $\mathrm{A} 1$ is present. Therefore, the right flank of the peak should be attributed to the disordered phase A1. This is the classical Zener peak already investigated in the past [25]. The maximum of the peak is close to the temperature where the A1 phase disappears. While Its amplitude dropped down going toward lower temperatures with the increase of the order. This drop is less abrupt at $0.5 \mathrm{~Hz}$, where the peak appears at lower temperatures. This observation shows that the Zener relaxation effectively falls down with the ordering when A1 disappears. Still, the AuCul and the AuCull phases allow the presence of a lower but visible peak $\mathrm{Pz}$, which is manifested as a low temperature shoulder. The Zener relaxation peak maximum is accompanied with an increase of the shear modulus and the total disappearance of $A 1$. In the area where there are two phases, the shear modulus presents fluctuations that accompany the disappearance of the small quantities of the orthorhombic phase AuCull before its stabilization when only phase $\mathrm{AuCul}$ is present. $\mathrm{P}_{\mathrm{GB}}$ represents the grain boundaries relaxation peak that appears adjacent to Zener peak at high temperature $[26,27]$ in A1. 


\section{Conclusion}

We assess the temperature-dependent phase transition sequence in a Au$25 \mathrm{wt} \% \mathrm{Cu}$ alloy upon heating and cooling from RT to $973 \mathrm{~K}$ when combining in-situ thermodiffraction experiments and mechanical spectroscopy at a sweep rate of 1 $\mathrm{K} /$ min. The initial state after the quenching is mainly a metastable cubic $A 1$ disordered phase but unavoidable nanograins of AuCul are present. The onset of the AuCul phase upon heating generates a transient peak together with a volume contraction. The AuCul phase shows a Zener peak, which suggests that a certain degree of disorder is present or at least appearance of the non thermal vacancies. The AuCull phase is characterized by a relaxation peak that should be attributed to the movement of antiphase boundaries. The transformation from AuCul to AuCull also leads to an expansion of the lattice. Upon cooling, the presence of all three possible phases is detected at the same time from 650 to $644 \mathrm{~K}$. Then, only AuCul and AuCull are present between 644 and $604 \mathrm{~K}$, before the stabilization of the tetragonal AuCul as the final state. This time the Zener peak seems to appear before the transition A1 ->AuCul+AuCull and partially disappears when only the AuCul phase is present. Therefore, a consistent part of the peak should be attributed to the copper relaxation in the disordered phase $\mathrm{A} 1$.

\section{Acknowledgments:}

Financial support from the Algerian Ministry of Higher Education and Scientific Research (PNE scholarship), Spanish Ministerio de Economía y Competitividad (MAT2016-78155-C2-1-R and FPI grant BES-2011-046948 to MSM.A.) and Gobierno del Principado de Asturias (GRUPIN14-060) are acknowledged. 


\section{References}

[1] C. Guoliang, X. Ni, T. Nsongo, Intermetallics. 12 (2004) 733-739.

[2] Y. Tanaka, K.I. Udoh, K. Hisatsune, K. Yasuda, Philos. Mag. A. 69.5 (1994) 925-938.

[3] B. Sprušil, B. Chalupa. Mater. Sci. Eng., A. 324 (2002) 58-61.

[4] D.W. Pashley, A.E.B. Presland. J. InstMetals. 87 (1958-1959), 419.

[5] D.W. Pashley, A.E.B. Presland, In: C.A. Neugebauer, J.B. Newkirk, D.A. Vermilyea, editors. Structure and properties of thin films New York: Wiley. (1959) 199-216.

[6] J. Sebor, J. sachl, V. Síma, Kovove Mater. 40 (2002) 5.

[7] P. Mašek, F. Chmelik, V. Šıma, A. Brinck, H. Neuhäuser, Acta Mater. 47 (1999) 427-434.

[8] J. Šachl, V. Šíma, W. Pfeiler, J. AlloysCompd. 378 (2004) 274-278.

[9] H.I. Aaronson, K. R. Kinsman, Acta Metall.25 (1977) 367-376.

[10] J.Bonneaux, M. Guymont, Intermetallics. 7 (1999) 797-805.

[11] R.Smith, J. S. Bowles, Acta Metall. 8 (1960) 405-415.

[12] A. J.Pedraza, J. Kittl, Acta Metall. 24 (1976) 835-843.

[13] I. S. Golovin, A. M. Balagurov, I. A. Bobrikov, V. V. Palacheva, J. Cifre, J. AlloysCompd. 675 (2016) 393-398.

[14] P. P. Fedorov, S. N. Volkov, Russ. J. Inorg. Chem. 61 (2016) 772-775.

[15] V. Šíma, Mater. Sci. Eng., A. 324 (2002) 62-67.

[16] I. S.Golovin, A. M. Balagurov, I. A. Bobrikov, J. Cifre, J. AlloysCompd.688 (2016) 310-319.

[17] I. S. Golovin, A. M. Balagurov, V. V. Palacheva, I. A. Bobrikov, V. Zlokazov, Mater. Des. 98 (2016) 113-119.

[18] J. Hennig, D. Mari, R. Schaller, Mater. Sci. Eng., A. 521 (2009) 47-51.

[19] J. Hennig, D. Mari, R. Schaller,Phys. Rev. B. 144 (2009) 116 - 79.

[20] A. K. Maier, I. Tkalcec, D. Mari, R. Schaller, Mater. Sci. Eng., A. 560 (2013) 466-472.

[21] A. K. Maier, I. Tkalcec, D. Mari, R. Schaller, Solid State Phenom. 184 (2012) 283-288. 
[22] A. K. Maier, I. Tkalcec, D. Mari, R. Schaller, Acta Mater. 74 (2014) 132-140.

[23] A. K. Maier, I. Tkalcec, D. Mari, R. Schaller, Scripta Mater. 66 (2012) 374-377.

[24] A. K. Maier, I. Tkalcec, D. Mari, R. Schaller, Acta Mater. 61 (2013) 6107-6113.

[25] C. Zener, Phys Rev. 8 (1947) 34-71.

[26] A. K. Maier, D. Mari, I. Tkalcec, R. Schaller, Mater. Sci. Eng., A. 632 (2015) 43-49.

[27] I. Tkalcec, D. Mari, R. Schaller, Arch. Metall. Mater. 60 (2015), 0353.

[28] A. Le Bail, Powder Diffraction. 20 (2005) 316-326.

[29] J. Rodríguez-Carvajal, RecentDevelopments of the Program FULLPROF, in Commission on Powder Diffraction (IUCr).Newsletter. 26 (2001) 12-19

[30] D.Hamana, L. Chetibi, L. Amiour, Phase Trans. 82 (2009) 755-766.

[31] Q.X. You, F.L. Yan, B. L. Xin, B. L. Xiao, J. P. Hong, Z.N. Yao, Transactions of Nonferrous Metals Society of China. 21 (2011) 1092-1104 